\title{
On the hunt for superhumans
}

$\mathrm{T}$ he future of preventive medicine may depend on harnessing one person's genetic boon to offset another's burden, say drug developers and geneticists.

Union Chimique Belge (UCB), a Belgian pharmaceutical company, recently launched a global competition to track down people with extraordinary physical traits and abilities, or phenotypes, such as immunity to certain diseases, low sensitivity to pain or the ability to heal quickly, in the hope of identifying genetic quirks that prevent disease.

The company is now reviewing submissions and will award prizes of up to $\$ 10000$ for the best entries describing groups or individuals with rare protective phenotypes.

A better understanding of the genetics behind these anomalies may lead to the discovery and development of new preventive medicines, says Dr. Duncan McHale, vice president of global exploratory development at UCB. "If there are people out there who have all the risk factors for type 2 diabetes, for example, but don't develop the disease, obviously there's something in their biology that's protecting them and could be used to protect others."

The approach is quickly gaining traction as genetic sequencing becomes more affordable, says Dame Kay Davies, director of the Medical Research Council's Functional Genomics Unit at the University of Oxford in England. "It's one of the big reasons that both big pharma and little pharma are interested in rare diseases now."

That interest is starting to pay off. Davies cites the recent development of an osteoporosis drug, now in late stage clinical trials, based on the study of

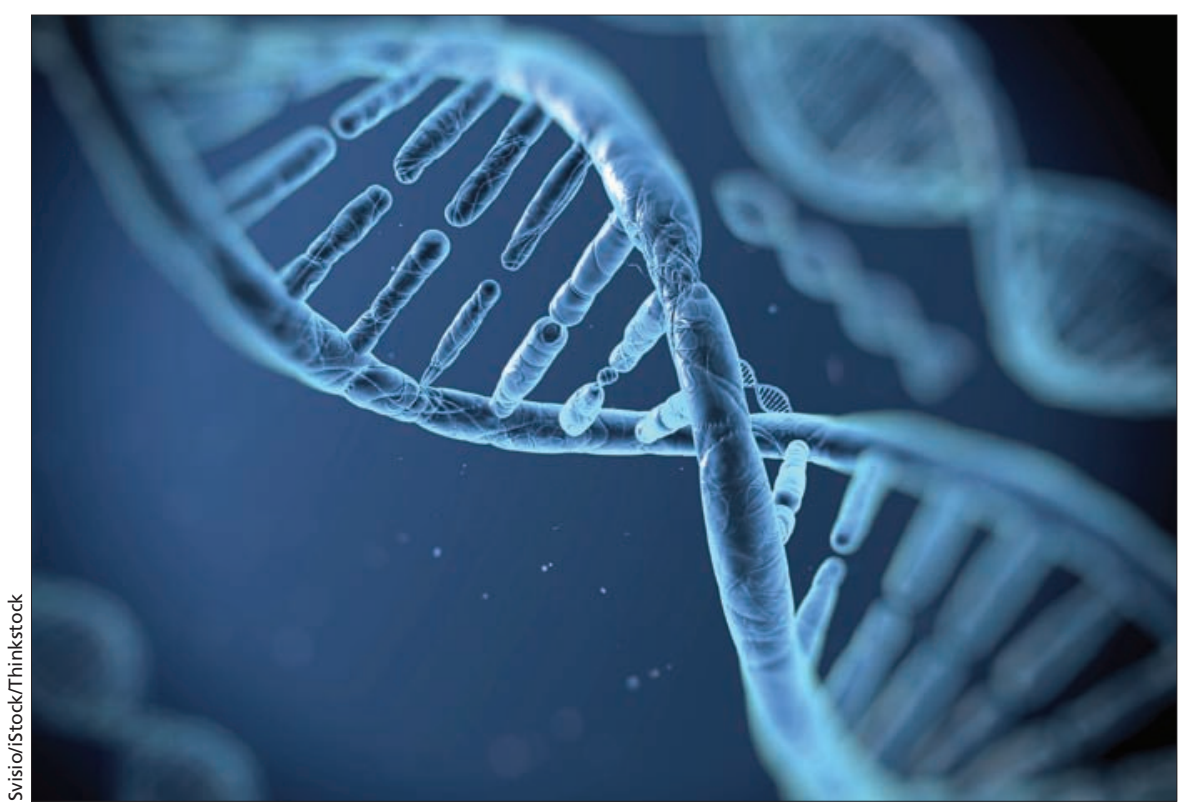

Understanding the genetic basis for extraordinary abilities, such as low sensitivity to pain or rapid healing, may lead to new preventive medicines.

three families in South Africa with unusually strong and dense bones.

"These extreme phenotypes can give us vital clues into new pathways of disease," she says.

According to Dr. Adam Shaw, general secretary of the British Society of Genetic Medicine, there are "potentially huge benefits" to directly studying humans to identify new drug targets, particularly for "complex human diseases that have no good animal models."

"Especially with dementia, one could significantly reduce the burden of disease with relatively subtle therapeutic effects, which could be difficult to recognize in mice," he says.

The trouble remains finding sufficiently extraordinary individuals to study.

"People with these protective phenotypes are simply less likely to come into contact with the health system or bio- medical research," explains McHale. Meanwhile, "you're looking for a really extreme variation from the norm because otherwise it's difficult to tease apart [a genetic cause]."

Enlisting the help of the general public to identify such individuals may be one solution, he says. But for now, crowd-sourcing efforts like UCB's competition are still largely relegated to pilot projects.

According to Davies, that may change as more people monitor their health using medical apps on their cellphones or computers. "Couple that with the power of genetics and there's a real opportunity here to identify people that appear to have abnormal readings on any of those parameters." - Lauren Vogel, CMAJ

CMAJ 2014. DOI:10.1503/cmaj.109-4762 